\title{
CORRELAÇÃO ENTRE TAXA METABÓLICA BASAL E VARIABILIDADE DA FREQUÊNCIA CARDÍACA NO TREINAMENTO RESISTIDO FUNCIONAL EM INDIVÍDUOS COM SÍNDROME METABÓLICA
}

\author{
Mariana Reis Jockner ${ }^{1}$, Allysiê Priscilla de Souza Cavina ${ }^{1,2}$, Altair Custódio Junior ${ }^{1,2}$, Jéssica Kirsch \\ Micheletti $^{1,2}$, Bruno Ryu Takahama ${ }^{1}$, Jayme Netto Junior ${ }^{1,2}$, Franciele Marques Vanderlei ${ }^{1,2}$ \\ ${ }^{1}$ Faculdade de Ciências e Tecnologia da Universidade Estadual Paulista - FCT/UNESP, ${ }^{2}$ Programa de Pós-Graduação em \\ Fisioterapia da FCT/UNESP, Presidente Prudente, São Paulo, Brasil. E-mail: franmvanderlei@gmail.com
}

\section{RESUMO}

O exercício físico é uma alternativa não farmacológica para agir sobre os indicadores da síndrome metabólica (SM). O treinamento resistido funcional (TRF) recruta maior número de grupos musculares o que pode acarretar maior gasto energético. Portanto, o objetivo desse estudo foi analisar a correlação entre a taxa metabólica basal (TMB) e a variabilidade da frequência cardíaca (VFC) no TRF em indivíduos com SM. O estudo foi um ensaio clínico não randomizado, composto de 32 indivíduos com SM que foram divididos em grupo treinamento resistido funcional (GTRF) e grupo controle (GC). A VFC foi coletada com um cardiofrequencímetro sendo selecionados os índices vagais e para a análise da TMB foi utilizado a bioimpedância. Foi observado correlação para o índice HF com a TMB somente antes do TRF. Portanto, não existe correlação significativa entre os índices vagais e a TMB em indivíduos com SM após a realização do TRF.

Palavras-chave: síndrome metabólica, exercício, sistema nervoso autônomo, metabolismo basal.

\section{CORRELATION BETWEEN BASAL METABOLIC RATE AND HEART RATE VARIABILITY IN FUNCTIONAL RESISTANCE TRAINING IN SUBJECTS WITH METABOLIC SYNDROME}

\begin{abstract}
Physical exercise is a non-pharmacological alternative to act on the indicators of the metabolic syndrome (MS). Functional resistance training (FRT), recruits a greater number of muscle groups, which can lead to greater energy expenditure. Therefore, the aim of this study was to analyze the correlation between the basal metabolic rate (BMR) and the heart rate variability (HRV) in FRT in individuals with MS. The study was a non-randomized clinical trial consisting of 32 subjects who had MS and were divided into functional resistance training group (FRTG) and control group (CG). The HRV was collected with a cardiofrequency meter, the vagal indices were selected and the analysis of BMR was performed by bioimpedance. Correlation was observed for the HF index with BMR only prior to FRT. Therefore, there is no significant correlation between vagal indices and BMR in individuals with MS after performing RFT.

Keywords: metabolic syndrome, physical exercise, autonomous nervous system, basal metabolism.
\end{abstract}

\section{INTRODUÇÃO}

A síndrome metabólica (SM) é o conjunto de alterações no metabolismo que geram disfunções sucessivas nos níveis lipídicos e glicêmicos, sendo a obesidade um de seus principais indicadores. As causas da SM podem estar associadas à fatores epigenéticos ou genéticos, o primeiro fator pode estar vinculado com o estilo de vida nocivo do indivíduo, incluindo a tríade do estresse, má alimentação e ausência de atividade física regular' ${ }^{1}$. Em decorrência da associação entre a SM com o estilo de vida do indivíduo, a atividade física se torna uma alternativa de destaque não farmacológica para agir sobre os seus indicadores $^{2}$. O treinamento resistido funcional (TRF) destaca-se como uma alternativa na intervenção da SM, sendo que a sua realização promove alterações metabólicas e fisiológicas ${ }^{3}$, como o aumento da taxa metabólica até 10 vezes 
maiores que os seus valores basais ${ }^{4}$ além de induzir uma modificação autonômica importante caracterizada por aumento da modulação parassimpática do indivíduo ${ }^{5}$.

O excesso de peso tem sido associado a um aumento nos marcadores inflamatórios e há um desequilíbrio no sistema nervoso autônomo (SNA), como diminuição da variabilidade da frequência cardíaca (VFC) ${ }^{6}$. A baixa VFC é frequentemente um indicador de adaptação anormal e insuficiente do SNA, o que pode indicar a presença de mau funcionamento fisiológico no indivíduo ${ }^{7}$, além disso, o estudo de Wolf et al. ${ }^{8}$ demonstra que a diminuição da VFC está correlacionada com o aumento da mortalidade e eventos arrítmicos em sobreviventes de infarto do miocárdio. No entanto, estudos indicam que o exercício físico induz uma modificação autonômica importante caracterizada por aumento da modulação parassimpática do indivíduo 9.

Desse modo, tem-se que o TRF pode ser considerado uma alternativa de tratamento não farmacológico para indivíduos com SM e que o mesmo pode ser capaz de promover o aumento da taxa metabólica basal e gerar adaptações autonômicas positivas faz-se importante estudar se os dois fatores podem estar correlacionados e desse modo utilizar de ferramentas simples para inferir o efeito sistêmico do treinamento em indivíduos com SM. Portanto, o objetivo do presente estudo foi correlacionar a taxa metabólica basal com a VFC no treinamento resistido funcional em indivíduos com SM.

\section{METODOLOGIA}

Trata-se de um ensaio clínico com amostragem por conveniência. A casuística foi composta de 32 indivíduos, de ambos os sexos, com idade entre 35 e 60 anos, que possuíam SM segundo as definições adotadas pela International Diabetes Federation (IDF). Os voluntários realizaram um programa a partir de modelos de exercícios funcionais por 16 semanas com frequência de três sessões semanais totalizando 39 sessões de treinos com intervalos recuperativos de 24 a 72 horas entre as sessões. O estudo foi apreciado e aprovado pelo Comitê de Ética da Faculdade de Ciências e Tecnologia FCT/UNESP sob número de parecer 026442/2017. Para a formulação de treinamento foi realizado o teste de 1 repetição máxima (1RM) e o Quadro 1 mostra a dinâmica de cargas, ou seja, semanas, sessões, número de séries e repetições, e intensidade de esforço. 
Quadro 1. Programa de treinamento resistido funcional periodizado.

\begin{tabular}{|c|c|c|c|c|}
\hline Fases & $\begin{array}{c}\text { Semana } \\
\text { s }\end{array}$ & Sessões & $\begin{array}{c}\text { Séries } \mathbf{x} \\
\text { Repetições }\end{array}$ & $\begin{array}{l}\text { Intensidade de esforço ( } \% \text { de } \\
\text { 1RM) }\end{array}$ \\
\hline \multirow{5}{*}{$\begin{array}{c}\text { Adaptativa } \\
\text { (Intensidade Leve) }\end{array}$} & 1 & $1 / 2 / 3$ & $2 \times 12$ & $30-40$ \\
\hline & 2 & $4 / 5 / 6$ & $2 \times 16$ & $30-40$ \\
\hline & 3 & $7 / 8 / 9$ & $2 \times 20$ & $30-40$ \\
\hline & 4 & $10 / 11 / 12$ & $2 \times 20$ & $30-40$ \\
\hline & 5 & \multicolumn{3}{|c|}{ Recuperativa } \\
\hline \multirow{5}{*}{$\begin{array}{l}\text { Intermediária } \\
\text { (Intensidade } \\
\text { Moderada) }\end{array}$} & 6 & $13 / 14 / 15$ & $1 \times 16 / 12 / 9$ & $40 / 50 / 60$ \\
\hline & 7 & $16 / 17 / 18$ & $1 \times 12 / 9 / 6$ & $50 / 60 / 70$ \\
\hline & 8 & $19 / 20 / 21$ & $1 \times 10 / 8 / 6$ & $60 / 70 / 80$ \\
\hline & 9 & \multicolumn{3}{|c|}{ Recuperativa } \\
\hline & 10 & $22 / 23 / 24$ & $1 \times 8 / 6 / 4$ & $70 / 80 / 90$ \\
\hline \multirow{6}{*}{$\begin{array}{c}\text { Avançada } \\
\text { (Intensidade Elevada) }\end{array}$} & 11 & $25 / 26 / 27$ & $\begin{array}{c}1 \times 6 / 4 / 2 / 4 / \\
6\end{array}$ & $80 / 90 / 100 / 90 / 80$ \\
\hline & 12 & $28 / 29 / 30$ & $\begin{array}{c}1 \times 6 / 4 / 2 / 2 / \\
4 / 6\end{array}$ & $80 / 90 / 100 / 100 / 90 / 80$ \\
\hline & 13 & $31 / 32 / 33$ & $\begin{array}{c}1 \times 6 / 4 / 2 / 2 / \\
2 / 4 / 6\end{array}$ & $\begin{array}{c}80 / 90 / 100 / 100 / 100 / 90 / \\
80\end{array}$ \\
\hline & 14 & \multicolumn{3}{|c|}{ Recuperativa } \\
\hline & 15 & $34 / 35 / 36$ & $\begin{array}{c}1 \times 6 / 4 / 2 / 2 / \\
2 / 4 / 6\end{array}$ & $\begin{array}{c}80 / 90 / 100 / 100 / 100 / 90 / \\
80\end{array}$ \\
\hline & 16 & $37 / 38 / 39$ & $\begin{array}{c}1 \times 6 / 4 / 2 / 2 / \\
2 / 4 / 6\end{array}$ & $\begin{array}{c}80 / 90 / 100 / 100 / 100 / 90 / \\
80\end{array}$ \\
\hline
\end{tabular}

Legenda: 1 RM = Repetição Máxima

Para a análise da VFC foram utilizados somente os índices vagais (rMSSD, HF $\left[\mathrm{ms}^{2}\right]$ e SD1 e a captação da $\mathrm{FC}$ foi realizada pelo cardiofrequencímetro da marca RS800cx. A taxa metabólica basal foi avaliada pela balança da marca TANITA, modelo BC - 418, Segmental Body Composition Analyzer, Iron Man/Inner Scaner de 8 canais.

\section{ANÁLISE ESTATÍSTICA}

Para a análise da correlação dos índices de VFC com a taxa metabólica basal foi utilizado o teste de correlação de Pearson ou Spearman de acordo com a normalidade dos dados. O limiar utilizado para avaliar quantitativamente as correlações foi baseado em Hopkins ${ }^{10}$ utilizando os seguintes critérios: 


\section{RESULTADOS}

Descritivamente, não foi observada diferença estatisticamente significante em relação aos índices vagais da VFC e a TMB nos momentos pré e pós-treinamento (Tabela 1; $p>0,05)$.

Tabela 1. Valores médios e respectivos desvios padrão dos índices vagais da VFC (rMSSD, HF [ms ${ }^{2}$ e SD1) e da TMB nos momentos pré e pós-treinamento.

\begin{tabular}{lll}
\hline Variáveis & Pré-Treinamento & Pós-Treinamento \\
\hline rMSSD $(\mathrm{ms})$ & $32,63 \pm 15,55$ & $30,17 \pm 17,22$ \\
$\mathrm{HF}\left(\mathrm{ms}^{2}\right)$ & $466,93 \pm 428,36$ & $395,62 \pm 392,41$ \\
$\mathrm{SD} 1(\mathrm{~ms})$ & $23,08 \pm 11,00$ & $21,40 \pm 12,26$ \\
$\mathrm{TMB}$ & $1729,43 \pm 317,47$ & $1729,87 \pm 365,37$ \\
\hline $\mathrm{TMB}=$ taxa metabólica basal, $\mathrm{ms}=$ milissegundos, $\mathrm{ms}^{2}=$ milissegundos ao quadrado.
\end{tabular}

Apenas para o índice $\mathrm{HF}\left(\mathrm{ms}^{2}\right)$ foi observada correlação com a TMB antes do treinamento resistido funcional com tamanho de efeito grande, ou seja, quanto maior a TMB pré maior a modulação vagal expressa pelo componente espectral (Tabela 2). Para os demais índices e momentos analisados não foi observada correlação significativa.

Tabela 2. Correlação entre a TMB e índices vagais da VFC (rMSSD, HF $\left[\mathrm{ms}^{2}\right]$ e SD1).

\begin{tabular}{|c|c|c|c|c|}
\hline \multirow{2}{*}{ Índices } & \multicolumn{2}{|c|}{ TMB Pré } & \multicolumn{2}{|c|}{ TMB Pós } \\
\hline & $R$ & $p$ & $\mathrm{R}$ & $P$ \\
\hline rMSSD (ms) & $\begin{array}{c}0,3996 \\
\text { (moderada) }\end{array}$ & 0,1252 & $\begin{array}{c}0,2817 \\
\text { (pequena) }\end{array}$ & 0,2904 \\
\hline $\mathrm{HF}\left(\mathrm{ms}^{2}\right)$ & $\begin{array}{c}0,6208 \\
\text { (grande) }\end{array}$ & $0,0103 *$ & $\begin{array}{c}0,4378 \\
\text { (moderada) }\end{array}$ & 0,089 \\
\hline $\mathrm{SD} 1$ (ms) & $\begin{array}{c}0,3998 \\
\text { (moderada) }\end{array}$ & 0,1250 & $\begin{array}{c}0,2854 \\
\text { (pequena) }\end{array}$ & 0,2840 \\
\hline
\end{tabular}

$\mathrm{TMB}=$ taxa metabólica basal, $\mathrm{ms}=$ milissegundos, $\mathrm{ms}^{2}=$ milissegundos ao quadrado, $\mathrm{R}=$ correlação .

\section{DISCUSSÃO}

O objetivo do estudo foi analisar a correlação entre a taxa metabólica basal e a VFC no treinamento resistido funcional em indivíduos com SM. Como resultado do estudo apenas no índice vagal $\mathrm{HF}\left[\mathrm{ms}^{2}\right]$ foi observada correlação de grande efeito com a TMB antes do treinamento resistido funcional, demonstrando que quanto maior a TMB pré maior a modulação vagal expressa pelo componente espectral. Para os demais índices e momentos analisados não foi observada correlação significativa, mostrando que uma variável é independente da outra e que, portanto, os sistemas energéticos e autonômicos devem ser avaliados separadamente para permitir conclusões fidedignas dos indivíduos com SM.

A obesidade central está associada a uma maior incidência de desenvolvimento de fatores de risco relacionados à DCV segundo estudo de Mehta et al. ${ }^{11}$ dentre eles a SM. Além disso, de acordo com o estudo de Farah et al. ${ }^{12}$ o sobrepeso pode estar associado a um aumento nos marcadores inflamatórios e ao desequilíbrio no sistema nervoso autônomo. Rossi et al. ${ }^{13}$ também verificaram diminuição da VFC devido a 
redução da modulação parassimpática e consequente predomínio relativo da modulação simpática em indivíduos com sobrepeso.

Nesse cenário, como forma de combater a obesidade, optar pela prática de exercício físico seria uma alternativa de prevenção e intervenção dos fatores da SM e melhora da VFC, pois possuem papel em proporcionar efeitos fisiológicos e metabólicos, tanto agudo quanto crônico, de uma maneira geral, como o aumento da massa muscular esquelética, diminuição dos estoques de gordura, aumento do gasto calórico, aumento da taxa metabólica de repouso, entre outros $^{14}$.

Entre os métodos de exercício físico se destaca o treinamento resistido, que segundo o estudo de Melby et al. ${ }^{15}$ gera uma taxa metabólica de repouso significativamente elevada até $15 \mathrm{~h}$ após o término da execução de sete exercícios contra-resistência, envolvendo três séries de 10-12RM com intervalos de dois minutos entre as séries.

Além de promover melhora na TMB o treinamento resistido também é capaz de alterar a modulação autonômica cardíaca melhorando a VFC. $O$ estudo de Barbosa et al. ${ }^{16}$ mostrou que o treinamento resistido funcional realizado de forma periodizada com duração de 12 semanas em indivíduos saudáveis, elevou os valores do índice vagal SD1 nas voluntárias do grupo treinado em relação ao grupo controle quando comparadas as diferenças obtidas entre os valores pré e pós-treinamento, sugerindo que o treinamento funcional promoveu aumento na modulação do ramo parassimpático do SNA.

Algumas limitações do estudo devem ser apontadas. A ausência do controle nutricional pode ser um fator que tenha influenciado na TMB e a VFC, visto que a diminuição da modulação autonômica cardíaca está particularmente ligada à gordura visceral ${ }^{17}$. Outra limitação do estudo é o fato de não ter sido um ensaio clínico randomizado, e os grupos comparados não serem homogêneos quanto à idade e sexo. Vale ressaltar que a randomização não foi possível por questões logísticas.

Apesar das limitações apresentadas, o estudo traz uma aplicabilidade clínica importante, pois o TRF foi capaz de proporcionar benefícios para os praticantes. De acordo com Vinik et al. ${ }^{18}$ um desequilíbrio entre a atuação do sistema nervoso simpático e parassimpático resulta em disfunção autonômica cardíaca que é um fator de Colloq Vitae 2018 set-dez; 10(3): 27-33. DOI: 10.5747/cv.2018.v10.n3.v240 ISSN 1984-6436/@ 2018 - Publicado pela Universidade do Oeste Paulista. Artigo Open Access sob uma licença CC BY-NC-ND (http://creativecommons.org/licenses/by-nc-nd/4.0/).

\section{CONCLUSÃO}

risco para doenças cardiovasculares. Sandercock e Brodi $^{19}$ relataram em seu estudo que a disfunção autonômica é prognóstica para vários desfechos de doenças cardiovasculares, incluindo morte súbita.

Tendo isso em vista, o presente estudo trás para a prática clínica que, por meio da aplicação de um TRF em indivíduos com SM não é possível obter resultados significantes ao correlacionar a TMB e os índices vagais da VFC. Mostra que uma variável é independente da outra e, portanto, os sistemas energéticos e autonômicos devem ser avaliados separadamente para permitir conclusões fidedignas dos indivíduos com SM.

Como perspectivas futuras para o estudo seria válido o acompanhamento nutricional dos voluntários com SM que realizassem o TRF, com a viabilidade em obter resultados significantes no momento pós-treinamento e resultados mais fidedignos entre a correlação da TMB e os índices vagais da VFC.

Não existe correlação entre os índices vagais da VFC e a taxa metabólica basal de indivíduos com SM que realizaram um programa de treinamento resistido funcional.

\section{CONFLITO DE INTERESSE}

Os autores declaram não haver qualquer potencial conflito de interesse que possa interferir na imparcialidade deste trabalho científico.

\section{REFERÊNCIAS}

1. Malambo $P$, Kengne AP, de Villiers A, Lambert $\mathrm{EV}$, Puoane T. Built environment, selected risk factors and major cardiovascular disease outcomes: a systematic review. PloS One. 2016;11(11):e0166846. DOI: https://doi.org/10.1371/journal.pone.0166846

2. Rennie KL, McCarthy N, Yazdgerdi S, Marmot $M$, Brunner E. Association of the metabolic syndrome with both vigorous and moderate physical activity. Int J Epidemiol. 2003;32(4):6006. DOI: https://doi.org/10.1093/ije/dyg179

3. Gustat J, Srinivasan SR, Elkasabany A, Berenson GS. Relation of self-rated measures of physical activity to multiple risk factors of insulin resistance syndrome in young adults: the 
Bogalusa Heart Study. J Clin Epidemiol. 2002;55(10):997-1006. DOI: https://doi.org/10.1016/S0895-4356(02)00427-4

4. Pereira Junior $M$, Andrade RD, Silveira FV, Baldissera UM, Korbes AS, Navarro F. Exercício físico resistido e síndrome metabólica: uma revisão sistemática. RBPFEX. 2013;7(42):529-39.

5. Cabral CC, Giacon TR, Campos EZ, Neto JG, Rodrigues B, Vanderlei LCM, et al. Impact of highintensity intermittent and moderate intensity continuous exercise on autonomic modulation in young men. Int J Sports Med. 2016;37(6):431-5. DOI: https://doi.org/10.1055/s-0042-100292

6. Miranda JMQ, Dias LC, Mostarda CT, Angelis $K D$, Figueira Junior AJ, Wichi RB. Efeito do treinamento de força nas variáveis cardiovasculares em adolescentes com sobrepeso. Rev Bras Med Esporte. 2014;20(2):125-30. DOI:

https://doi.org/10.1590/1517-

$\underline{86922014200201703}$

7 Pumprla J, Howorka K, Groves D, Chester M, Nolan J. Functional assessment of heart rate variability: physiological basis and practical applications. Int J Cardiol. 2002;84(1):1-14. DOI: https://doi.org/10.1016/S0167-5273(02)00057-8

8 Wolf MM, Varigos GA, Hunt D, Sloman JG. Sinus arrhythmia in acute myocardial infarction. Med J Aust. 1978;2(2):52-3.

9. Cabral CC, Giacon TR, Campos EZ, Neto JG, Rodrigues B, Vanderlei LCM, et al. Impact of highintensity intermittent and moderate intensity continuous exercise on autonomic modulation in young men. Int J Sports Med. 2016;37(6):431-5. DOI: https://doi.org/10.1055/s-0042-100292

10. Hopkins WG. Measures of reliability in sports medicine and science. Sports Med. 2000;30(1):115. DOI: https://doi.org/10.2165/00007256200030010-00001

11. Mehta RK. Impactos da obesidade e estresse no desenvolvimento da fadiga neuromuscular e variabilidade da frequência cardíaca associada. Int J Obes. 2015;39:208-13. DOI: https://doi.org/10.1038/ijo.2014.127

12. Farah BQ, Prado WLd, Tenório TRdS, Ritti-Dias RM. Relação entre variabilidade da frequência cardíaca e indicadores de obesidade central e geral em adolescentes obesos normotensos. Colloq Vitae 2018 set-dez; 10(3): 27-33. DOI: 10.5747/cv.2018.v10.n3.v240 ISSN 1984-6436/C 2018 - Publicado pela Universidade do Oeste Paulista.

Artigo Open Access sob uma licença CC BY-NC-ND (http://creativecommons.org/licenses/by-nc-nd/4.0/).
Einstein (São Paulo). 2013;11(3):285-90. DOI: https://doi.org/10.1590/S1679$\underline{45082013000300005}$

13. Rossi RC, Vanderlei LCM, Gonçalves ACCR, Vanderlei FM, Bernardo AFB, Yamada KMH, et al. Impact of obesity on autonomic modulation, heart rate and blood pressure in obese young people. Auton Neurosci. 2015;193:138-41. DOI: https://doi.org/10.1016/i.autneu.2015.07.424

14. Meirelles CM, Gomes PSC. Efeitos agudos da atividade contra-resistência sobre $\mathrm{o}$ gasto energético: revisitando o impacto das principais variáveis. Rev Bras Med Esporte. 2004;10(2):12230. DOI: $\quad$ https://doi.org/10.1590/S1517$\underline{86922004000200006}$

15. Melby C, Scholl C, Edwards G, Bullough R. Effect of acute resistance exercise on postexercise energy expenditure and resting metabolic rate. J Appl Physiol. 1985;75(4):184753.

DOI:

https://doi.org/10.1152/jappl.1993.75.4.1847

16. Barbosa MPCR, Júnior JN, Cassemiro BM, Souza NM, Bernardo AFB, Silva AKF, et al. Impact of functional training on cardiac autonomic modulation, cardiopulmonary parameters and quality of life in healthy women. Clin Physiol Funct Imaging. 2016; 36:318-25. DOI: https://doi.org/10.1111/cpf.12235

17. Durstine JL, Haskell WL. Effects of exercise training on plasma lipids and lipoproteins. Exerc Sports Sci Rev. 1994;22:477-521. DOI: https://doi.org/10.1249/00003677-19940100000017

18. Vinik ai, maser re, ziegler d. neuropathy: the crystal ball for cardiovascular disease? Diabetes Care. 2010;33(7):1688-9. DOI: https://doi.org/10.2337/dc10-0745

19. Sandercock GR, Brodie DA. The role of heart rate variability in prognosis for different modes of death in chronic heart failure. Pacing Clin Electrophysiol. 2006;29(8):892-904. DOI: https://doi.org/10.1111/j.15408159.2006.00457.x 
Recebido para publicação em 16/08/2018

Revisado em 23/08/2018

Aceito em 31/08/2018 